\title{
Special Recommendations in Expanding the Roles of Pharmacists in Response to COVID-19 Pandemic in Low and Middle-Income Countries (LMICs)
}

\author{
Muhammad Ahmer Raza ${ }^{1,2}$, Shireen Aziz ${ }^{3,4}$, Shahid Masood Raza ${ }^{1,4,5 *}$ and Sana Shahzad ${ }^{1,4}$ \\ ${ }^{1}$ Faculty of Pharmacy, The University of Faisalabad, Pakistan \\ ${ }^{2}$ Department of Clinical Pharmacy, School of Pharmaceutical Sciences, Shandong University, China \\ ${ }^{3}$ School of Pharmacy, Zhengzhou University, China \\ ${ }^{4}$ Faculty of Pharmacy, University of Sargodha, Pakistan \\ ${ }^{5}$ School of Pharmacy, Tongji Medical College, Huazhong University of Science and Technology, China \\ *Corresponding author: Shahid Masood Raza, School of Pharmacy, Tongji Medical College, Huazhong University of Science and \\ Technology, Wuhan 430030, Hubei, China, Email: shahipharmacist@gmail.com
}

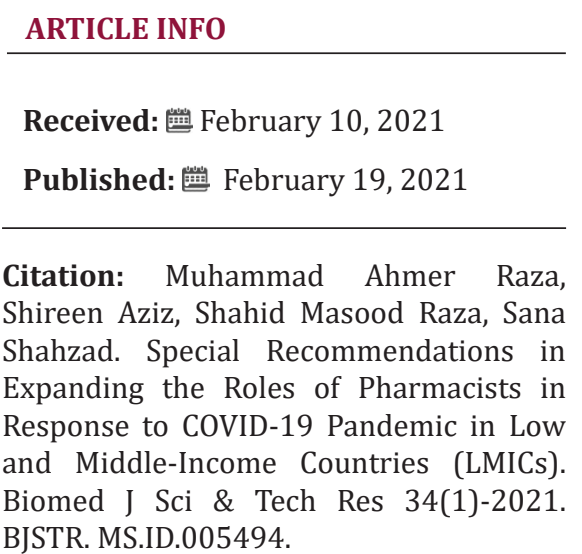

\section{ABSTRACT}

The coronavirus disease in 2019 (COVID-19) is an ongoing pandemic caused by severe acute respiratory syndrome coronavirus 2 (SARS-CoV-2). The COVID-19 pandemic has greatly affected the functioning of the healthcare settings. More people are hospitalizing, and mortality rate is rising due to overcrowded hospitals and staff shortages. Numerous studies have been published in the Europe and United States (U.S) for the legal extensions in the roles of pharmacists in response to COVID-19 global pandemic. However, novel responsibilities have not been addressed yet for low and middle-income countries (LMICs).

Keywords: COVID-19; SARS-CoV-2; Pharmacist; LMICs

\section{Introduction}

When lockdowns were common worldwide, pharmacies are an example that has remained open to serve the public during these trying times. Pharmacists answer questions for patients regarding their health on a daily basis, whether it is on the phone about medications or in person when someone is experiencing symptoms. As the burden on healthcare workers increasing during this pandemic, pharmacists can help reduce it. With pharmacists being the most accessible health care providers, it is easy to make possible regarding the expansion of their role during and after the COVID-19 pandemic $[1,2]$. With the unavailability of a specific antiviral or a vaccine, non-pharmaceutical interventions could be benchmark in curbing an ongoing COVID-19 pandemic. Pharmacists can play a very important role since they interact directly with patients. Some countries are implementing laws that are allowing pharmacists to expand their roles temporarily in response to COVID-19 pandemic, but these laws vary by country to country $[3,4]$. However, there are no such recommendations in expanding the roles of pharmacists in LMICs in response to the COVID-19 pandemic. Thus, the purpose of this article is to offer special recommendations for the legal extension in the roles of pharmacists in the light of COVID-19 pandemic in the LMICs by considering recent breakthroughs in the role of pharmacists in the European countries and the U.S. This article will enable pharmacists of the LMICs actively join in the fight against the ongoing COVID-19 pandemic.

\section{Special Recommendations}

The special recommendations in expanding the role of pharmacists in the light of COVID-19 pandemic in LMICs shown in Figure 1 and should include the following: 


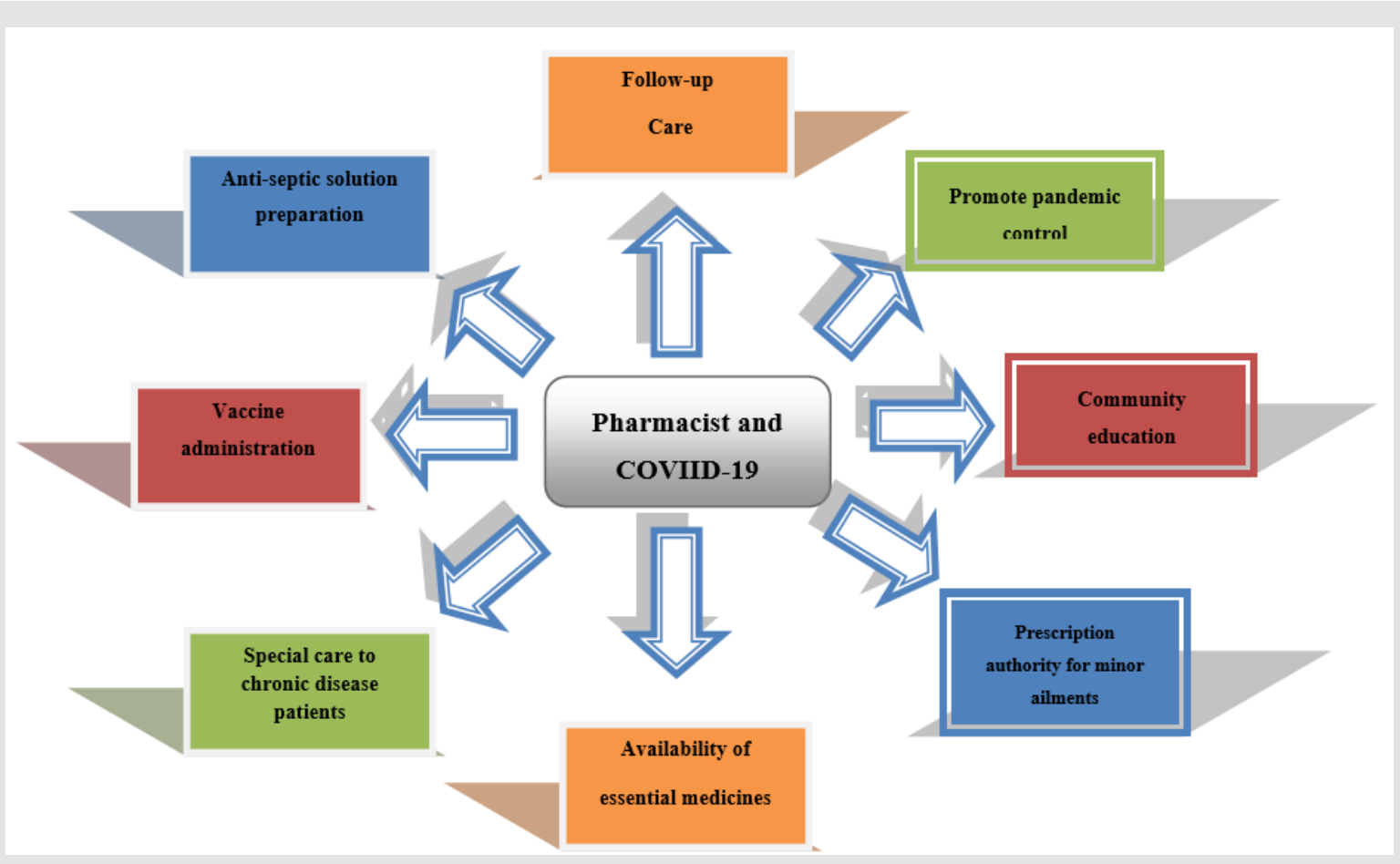

Figure 1: Special recommendation in expanding the roles of pharmacists in the light of COVID-19 pandemic in LMICs.

a) Promote pandemic control

b) Community education

c) Prescription authority for the management of minor aliments

d) Special care to chronic disease patients

e) Availability of essential medicines for COVID-19 patients

f) Vaccine administration with nurses

g) Preparation of anti-septic solutions

h) Follow-up care

\section{Discussion}

The role of pharmacists in community pharmacies and hospital settings can be seen during COVID-19 pandemic in developed countries. As stated in this article, an extensive range of new responsibilities have been introduced in the Europe and the U.S to expand the legal roles of the pharmacists to fight an ongoing pandemic $[3,4]$. The pharmacist's contribution has been appreciated by the New Zealand government by additional salary for their support. Hotline numbers have been issued to encourage phone consultation and prescription to reduce public visits [5]. Pharmacists in Australia are receiving prescriptions through mails/faxed/email, and phone messages for home delivery services [6]. Pharmacists in LMICs can play an integral role in promoting pandemic control by implementing guidelines designed for ensuring patient and staff safety. Pharmacists can promote social distancing by organizing pharmacies in such a way as to make it difficult for the virus to spread. Pharmacists can design temporary barriers to limit the number of patients in the pharmacy at any given time and to increase the distance between patients.

These actions should be considered significant due to an increasing number of COVID-19 patients in the second wave of pandemic. Furthermore, pharmacist interacts directly with patients. They can play an important role in educating people and patient on how to behave in the pharmacy and, how patients can protect themselves from infection. Basic guidance on COVID-19 symptoms and transmission routes, provided by pharmacists to help the community population understand the pandemic situation properly and to promote early identification of suspected individuals. Pharmacists can regularly provide information to community and patients about international and local healthcare recommended guidelines. This can also help to improve adherence and, consequently, patient safety.

In response to an ongoing pandemic, governments have expanded legal responsibilities of pharmacists in the area of prescribing for minor ailments. It is worth mentioning the example of Poland, in which pharmacists' prescribing authority has been expanded recently [7]. Pharmacists can prescribe drugs for themselves and their relatives to reduce workload on doctors and physicians in the light of COVID-19 pandemic. This novel responsibility can be a game changing for LMICs where doctors' shortage has remained an important issue. By giving the authority of prescribing drugs in managing minor ailments to pharmacists in LMICs means that doctors and physicians can focus their skills 
where they are most needed in the ongoing pandemic, for example on diagnosing and treating patients with more complex conditions.

Medicine shortage was one of the main challenging problems during the start of COVID-19 pandemics. Pharmacists can play an important role in overcoming this problem by ensuring the availability of essential medicines by changing the same medicine with different strength, generic substitution, therapeutic substitution, preparing compounded formulation and importing the medicine from another country or by purchasing the same medicine from alternative authorized sources (nationally). Pharmacists can also play an important role in managing the temporal shortage of antiseptics by compounding antiseptics. This additional responsibility would be a novel step towards minimizing the further spread of pandemic.

The released data of Centers for Disease Control (CDC) and Prevention report highlights the dangers of chronic diseases with COVID-19 infection. Death rates are 12 times higher for COVID-19 patients with chronic illnesses than for others who become infected [8]. Pharmacists are a community-based knowledge resource that can help people understand the dangers of chronic disease and the importance of prevention. Pharmacists work with other members of the healthcare team and can refer patients' chronic disease related issues to them. They can also provide additional information to COVID-19 patients having associated chronic diseases and educate them to perform self-monitoring. In addition, introducing the permission of vaccine administration by a pharmacist can reduce workload on nurses. For this, campaigns related to community pharmacy-based immunization can play an important part in the LMICs.

COVID-19 disease caused by the SARS-CoV-2 virus affect multiple organs in the body. Therefore, long-term follow-up care by pharmacists is a real need of time. Pharmacist can assess the health status of COVID-19 patients by phone call, phone message, video call and email, and, if necessary, refer patients for followup treatment with another health care provider. Pharmacists may ask laboratory tests of COVID-19 patients to assess their health status or to determine whether a medication is working safely and effectively. Depending on the result of the health status assessment, the pharmacist may recommend medication adjustments or lifestyle changes.

As SARS-CoV-2 virus affect multiple organs, COVID-19 patients move through the healthcare system, they receive care from different specialties in a variety of settings and from many members of the healthcare team including chest specialists, neurologists, hematologists, cardiologist, psychologists, and physical therapists. Any time a patient is transferred from one care setting to another there is a potential for medication-related errors to occur. Factors such as poor communication among the healthcare team and low access to patient records often result in high rates of avoidable medication-related problems. Common errors during care transitions include a medication accidently being discontinued or more than one medication being prescribed to treat the same condition. Pharmacists during follow-up care of COVID-19 patients can guide medical doctors and general practitioners (GPs) about the patient's complete medication list. This additional responsibility can have positive impact on COVID-19 infected patient safety by managing care transitions.

\section{Conclusion}

In LMICs, the scarcity of resources is a challenge with regard to the prevention and management of COVID-19. In conclusion, by expanding the roles of pharmacists in LMICs (where sources are limited) can help in controlling the spread of pandemic by promoting education and pandemic control activities by pharmacists, reducing mortality rates by giving them additional and novel responsibilities to overcome the burden of doctors and nurses. The further expansion of services depends on the severity and the duration of the pandemic.

\section{Ethics Approval and Consent to Participate}

Not applicable.

\section{Consent for Publication}

All authors approved the manuscript.

\section{Availability of Data and Materials}

Not applicable.

\section{Competing Interests}

All authors declare no competing interests.

\section{Funding}

None.

\section{Author Contributions}

MAR and SA were engaged in reviewing the literature, writing, designing the model, and drafting the manuscript. SS and SMR were responsible for the conception and critical revision of the manuscript. All authors have read and approved the final text of the manuscript.

\section{Acknowledgment}

We are thankful to our colleagues to address the attention to this issue.

\section{References}

1. Hedima EW, MS Adeyemi, NY Ikunaiye (2021) Community pharmacists: On the frontline of health service against COVID-19. Research in Social and Administrative Pharmacy 2020.

2. Song Z, Yang Hu, Siqian Zheng, Li Yang, Rongsheng Zhao, et al. (2020) Hospital pharmacists' pharmaceutical care for hospitalized patients with COVID-19: Recommendations and guidance from clinical experience. Research in Social and Administrative Pharmacy 2020. 
3. Merks P, Marta Jakubowska, Ewelina Drelich, Damian Swieczkowski, Joanna Bogusz, et al. (2021) The legal extension of the role of pharmacists in light of the COVID-19 global pandemic. Research in Social \& Administrative Pharmacy.

4. (2020) Expansion of community pharmacy activties in relation to COVID-19.

5. (2021) Pharmaceutical Society of New Zealand (Inc.). COVID-19: Guidance Provided By The Society For Pharmacy.

6. (2020) Australian Government Department of Health. Fact SheetCoronavirus (COVID-19) Health Plan, Primary Care-Home Medicine Services Response to COVID-19.

\section{ISSN: 2574-1241}

DOI: 10.26717/BJSTR.2021.34.005494

Shahid Masood Raza. Biomed J Sci \& Tech Res

(C) (P) This work is licensed under Creative Submission Link: https://biomedres.us/submit-manuscript.php
7. (2021) Dossier. Coronavirus.

8. (2020) Journal of Emergency Services. Coronavirus Death Rate is Higher for Those with Chronic Illnesses.

$\begin{array}{ll}\text { BIOMEDICAL } & \text { Assets of Publishing with us } \\ \text { RESEARCHES } & \text { - Global archiving of articles } \\ \text { - Immediate, unrestricted online access }\end{array}$

